\title{
Impact of Dredging on the Fisheries of Igbedi Creek, Upper Nun River, Niger Delta, Nigeria
}

\author{
${ }^{1}$ Seiyaboh, E.I., Ogamba, E.N., ${ }^{2}$ Utibe, D.I. and ${ }^{3}$ Sikoki, F.D. \\ ${ }^{I}$ Department of Biological Sciences, Faculty of Science, Niger Delta University, Wilberforce Island, Yenagoa, \\ Bayelsa State. \\ Department of Animal \& Environmental Biology, University of Port Harcourt, PMB 53223, Port Harcourt, \\ Nigeria, \\ ${ }^{3}$ Centre for Marine Pollution Monitoring and Seafood Safety, University of Port Harcourt, PMB 5323, Port \\ Harcourt, Nigeria.
}

\begin{abstract}
The impact of dredging on the fisheries of Igbedi Creek, upper Nun River, Niger Delta was investigated from June 2009 to May 2011. Two locations - Ogobiri (Dredged) and Agoro-Gbene (Un-dredged) were studied. A total of 26,988 specimens representing 22 species belonging to 13 families were caught at Ogobiri (Dredged) Location; while a total of 33,275 representing 28 species belonging to 16 families were caught at Agoro-Gbene (Un-dredged) Location during the sampling period. There was a decrease in the number of fish species recovered in the undredged from 28 to only 22 in the dredged area. Fish from the dredged area exhibited negative allometric growth with length exponent (b) ranging from $0.911 \pm 0.04$ (Brycinus macrolepidotus). The only exception was Schulbe uranoscopus, which exhibited isometric growth with length exponent " $b$ " $=3.027 \pm 0.03$. In the undredged location, three species, exhibited isometic growth (Synodontis batensodath ( $b=3.095 \pm 0$-07); Schilbe uranoscopus $(b=3.097 \pm 0.03)$ and Distichodus faciatus $(b=3.021 \pm 0.11)$. The other species exhibited negative.allometric growth with length exponents ranging from $0.777 \pm 0.004$ to $2.560 \pm 0.04$ with the exception of Hepsetus odoe which exhibited positive allometic growth $(b=3.412 \pm 0.07)$. Correlation coefficients between length and weight " $r$ " ranged from 0.739 (Eleoties senegalensis) to 0.978 (Schulbe mystus) in the dredged; and between 0.447 (Polypterus ansorgi) to 0.994 (synodontis membranoceous) in the undredged locations. Student's t-test values at $95 \%$ level showed significant differences in condition factor between the two areas. These results are clear indications that dredging significantly affected the fisheries of the creek.
\end{abstract}

\section{Introduction}

Dredging is a process that involves the excavation of water beds to remove sediments, pollutants, shellfish and other materials. The methods and machinery used in dredging vary widely. Most dredging is done by ships that tow a dredge along the water bed. Self standing dredges and dredge pumping stations are used for routine tasks. A dredge, which is the catch all term for the different types of machinery that perform dredging, can cut away sediment, scoop materials out like a back hoe or suction them through a large pipe to be deposited into a ship.

The effects of dredging on aquatic organisms have been a source of environmental concern for several decades. One category of concern that has frequently arisen in connection with projects involving dredging for navigational purposes deals with mortality of fish and shellfish entrained during the dredging process (Reine and Clark, 1998).

Dredging and related activities could disrupt fisheries and/or damage spawning grounds which may also have deleterious impacts on key fishery resources and, to some extent, the fishing industry (Ault et al., 1998; Gebhards, 1973).

Specifically, appropriately designed studies to address dredging impacts are very limited and the lack of relevant data continues to foster controversy on dredging impact assessments. Until adequate data are available, quantifying biological responses to the potential dredging-induced impacts must unfortunately remain subjective (Clark, 1979). Furthermore, for decades the effects of dredging on aquatic resources have been an issue of increasing environmental concern. However, very little work has been done to detect the effects of dredging at population-level of mobile epi-benthic macro-invertebrates and dermal fishes (Ault et al., 1998).

This research is intended to access the level of impact of dredging operations on the fisheries of Igbedi Creek, upper Nun River. It is intended to stress that in dredging operations particular attention should be paid to the reduction of the vulnerability of the environment and those species of organisms (fisheries in this case) most vulnerable to the risks associated with such operations. Basically, this research looked at some aspects of the fisheries of Igbedi Creek, upper Nun River in a Dredged site as compared to an Un-dredged site.

The objectives of the research are:

- To assess the fish species distribution in the Dredged and Un-dredged sites. 
- To assess the Length and Weight relationship of the fish species in the Dredged and Un-dredged sites and determine Fish Assemblage; Catch Composition; Relative Abundance and Condition factor.

- Compare the data obtained in the Dredged and Un-dredged site and determine the possible impact of dredging on fisheries of Igbedi Creek, upper Nun River.

The study is expected to reveal the impact on the ecosystem as direct consequences of dredging operations in Igbedi Creek, upper Nun River using fish as an indicator. Fisheries impact is a significant conclusion for environmental and ecological change in aquatic systems (Karr, 1981).

\section{[1]Study Area}

\section{Methodology}

The study was carried out in Igbedi Creek, a tributary of the Upper Nun River in the Niger Delta located between latitude $5^{0} \mathrm{~N} 01^{1}$ and $6^{0} 17^{1} \mathrm{E}$. The stretch of the river is a long and wide meander whose outer concave bank is relatively shallow with sandy point bars (Abowei, 2000). The depth and width of the river vary slightly at different points (Sikoki et. al., 1998). The minimum and maximum widths are 200 and 250 meters respectively. The river is subjected to tidal influence in the dry season. Water flows rapidly in one direction during the flood (May - October). At the peak of the dry season, the direction of flow is slightly reversed by the rising tide. At full tide the flow is almost stagnant.

\section{[2]Field Study \& Collection of Samples}

Two communities were chosen as sample communities for this study. These are: Ogobiri (Dredged Location), in the Sagbama Local Government Area (SALGA) i.e. the community where the dredging is taking place, and Agoro-Gbene (Un-dredged Location), also in Sagbama Local Government Area (SALGA) which is a community that is relatively undisturbed. Both communities are located along the igbedi creek, upper Nun River and are both predominantly fishing communities.

For the purpose of this study, the selected dredged and non-dredged sites were divided into stations: Ogobiri $\mathrm{OGO}_{1} \mathrm{DA}=$ Dredged Area, $\mathrm{OGO}_{\mathrm{I} \& 2} \mathrm{DS}=$ Downstream, $\mathrm{OGO}_{1 \& 2} \mathrm{US}=$ Upstream; Agoro-gbene $\mathrm{AGO}_{1} \mathrm{DS}=$ Downstream, $\mathrm{AGO}_{1} \mathrm{MD}=$ Midstream, $\mathrm{AGO}_{1} \mathrm{US}=\mathrm{Upstream}$.

\section{Collection of Fish Samples}

Sampling was carried out forth nightly between June 2009 and May 2011 using gillnets, long lines, traps and stakes. The researcher fished for himself and also employed the services of fishers for sampling. Catches were isolated and conveyed in thermos cool boxes to the laboratory on each sampling day. Fish specimens were identified using monographs, descriptions, checklists and keys (Daget, 1954; Boseman, 1963; Reed et. al., 1967; Holden and Reed, 1972; Poll, 1974; Whyte, 1975; Jiri, 1976; Reed \& Sydenham, 1978; Otobo, 1981; Alfred Ockiya, 1983; Whitehead, 1984; Loveque et al., 1991.

Total length and weight of fish specimens were measured to the nearest centimetre and grammes respectively, to obtain data for length-weight relationship. The total length (TL) of the fish was measured from the tip of mouth to the caudal fin using meter rule calibrated in centimetre. Fish samples were measured to the nearest centimetre. The weight of each fish was obtained after draining water from the buccal cavity and blot drying samples with a piece of clean hand towel. Weighing was done with a tabletop weighing balance to the nearest gram.

\section{Length - Weight Relationship}

The relationship between the length (L) and weight (W) of the various fish species were expressed by the exponential equation (Pauly, 1983):

$$
\mathrm{W}=\mathrm{aL}^{\mathrm{b}}
$$

Where

$\mathrm{W}=$ Weight of fish in $(\mathrm{g})$

$\mathrm{L}=$ Total Lenght of fish in $(\mathrm{cm})$

$\mathrm{a}=$ Constant (intercept)

$b=$ The Length exponent (Slope)

The "a" and " $b$ " values were obtained from a linear regression of the length and weight of fish. The correlation (r) that is the degree of association between the length and weight was computed from the linear regression analysis.

\section{Condition Factor}

The condition factor $(\mathrm{K})$ of the experimental fish was estimated from the relationship:

Where;

$$
\mathrm{K}=100 \mathrm{~W} / \mathrm{L}^{3} \quad \text { (Eqn. 2) }
$$


$\mathrm{K}=$ Condition Factor

$\mathrm{W}=$ Weight of Fish $(\mathrm{g})$

$\mathrm{L}=$ Length of Fish $(\mathrm{cm})$

\section{[3]Analysis of Experimental Data}

The following statistical tools were used to analyzed the data obtained - Regression and Correlation Analysis (RECA) for linear regression of length and weight of fish, Microsoft Excel (2010) for computation of means and standard deviation; Statistical Package for Social Sciences (SPSS) and FISAT (Gayando and Pauly, 1997) for descriptive statistics, length-weight relationship and condition factor of fish.

\section{Results And Discussion}

A total of 26,988 specimens representing 22 species belonging to 13 families were caught at Ogobiri (Dredged) Location; while a total of 33,275 representing 28 species belonging to 16 families were caught at Agoro-Gbene (Un-dredged) Location during the sampling period. A total of 60,263 specimens were obtained from both locations. In Ogobiri Location, Polydactylus quadrifilis was the most abundant in biomass $(85.73 \mathrm{Kg})$ accounting for $8.6 \%$ of the total weight of fish specimens caught. Schilbe uranoscopus was the most numerous (2021) accounting for $7.5 \%$ of the total number of fish specimens caught. In Agoro-Gbene location, Distichodus brevipinnis had the highest biomass $(206.76 \mathrm{Kg})$ accounting for $9.5 \%$ of the total weight of fish specimens caught. Schilbe uranoscopus was the most numerous (2245) accounting for $6.8 \%$ of the total number of fish specimens caught. The fish species with the least percentage composition in biomass in Ogobiri location was Petrocephalus bovei $(7.60 \mathrm{Kg})$ accounting for $0.8 \%$ of the total weight of fish specimens caught; and Agoro-Gbene location it was Parallia pellucida $(7.66 \mathrm{Kg})$ accounting for $0.4 \%$ of the total weight of fish specimens caught. In Ogobiri location, Hepsetus odoe was the least numerous (1012) accounting for $3.8 \%$ of the total number of fish specimens caught; while in Agoro-Gbene location, Cynoglossus senegalensis was the least numerous (828) accounting for $2.5 \%$ of the total number of fish specimens caught. Tables $3 \& 4$ show fish species caught and their composition in Ogobiri (Dredged) \& Agoro-Gbene (Un-dredged) locations (June 2009 - May 2011). The result of the respective percentage composition in number and total weight of fish caught in the various locations showed that the following: Ogobiri (Dredged) location - 26,988 (44.8\%); 1000.57 (31.5\%) and Agoro-Gbene Un-dredged) location - 33,275 (55.2\%); 2175.51 (68.5\%). This study revealed that Agoro-Gbene location recorded the highest values both in total number and weight of $55.2 \%$ and $68.5 \%$ respectively. This may be attributed to the relatively better environmental condition in Agoro-Gbene (Un-dredged) location as revealed by this investigation when compared to Ogobiri (Dredged) location, which may be a direct consequence of the dredging in this location. The values obtained in Agoro-Gbene (Un-dredged) location are significantly higher $(\mathrm{p}<0.05)$ than those obtained in Ogobiri (Dredged) location.

Table 1: Fish Species caught and their Composition in Ogobiri (Dredged) Location (June 2009 - May 2011)

\begin{tabular}{|c|c|c|c|c|}
\hline Family/Species & No. of Specimens & $\%$ No. of Specimen & $\begin{array}{c}\text { Weight (Kg) of } \\
\text { Specimens }\end{array}$ & $\begin{array}{r}\text { \% Weight } \\
\text { Specimen } \\
\end{array}$ \\
\hline $\begin{array}{l}\text { CHARACIDAE } \\
\text { Bracinus nurse }\end{array}$ & & & & \\
\hline BDycinus macrolepidotus. & 1025 & 3.8 & $\begin{array}{l}31.00 \\
79.92\end{array}$ & 8.0 \\
\hline 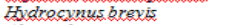 & 1227 & 4.6 & 74.77 & 7.5 \\
\hline $\begin{array}{l}\text { Alestess baremose } \\
\text { MONOCHOKIDAE }\end{array}$ & 1048 & 3.9 & 33.36 & 3.3 \\
\hline Synedontis batenseda & 1145 & 4.2 & 30.24 & 3.0 \\
\hline $\begin{array}{l}\text { Synodontis membranaceozs. } \\
\text { BA GRIDAE }\end{array}$ & 1211 & 4.5 & 18.20 & \\
\hline Chrovichsthos furcatus. & 1204 & 4.5 & 69.70 & 7.0 \\
\hline $\begin{array}{l}\text { Clargotes laticepge } \\
\text { POL YNEMIDAE }\end{array}$ & 1196 & 4.4 & 45.00 & 4.5 \\
\hline $\begin{array}{l}\text { Polydactaluss augadxiftis } \\
\text { CLUPEIDAE }\end{array}$ & 1186 & 4.4 & 85.73 & 8.6 \\
\hline Odgaxa thrissa mente & 1144 & 4.2 & 14.60 & 1.5 \\
\hline $\begin{array}{l}\text { CYNoGLOOSSIDAE } \\
\text { Cyneglossus senegaleusis. } \\
\text { CYPRINID AE }\end{array}$ & 1258 & 4.7 & 57.54 & 5.8 \\
\hline $\begin{array}{l}\text { CYPRINIDAE } \\
\text { Laber Goubie }\end{array}$ & 1232 & 4.6 & 62.89 & 6.3 \\
\hline $\begin{array}{l}\text { Enber senegralensas } \\
\text { MORMYRIDAE }\end{array}$ & 1286 & 4.8 & 57.90 & 5.8 \\
\hline $\begin{array}{l}\text { MORMYRIDAE } \\
\text { Gnathenemus abadij }\end{array}$ & 1210 & 4.5 & $\begin{array}{c}36.77 \\
760\end{array}$ & 3.7 \\
\hline $\begin{array}{l}\text { Petrecephatus bevei } \\
\text { DISTICHODONTIDAE }\end{array}$ & 1036 & & 7.60 & 0.8 \\
\hline $\begin{array}{l}\text { Distichedlus brexipinghis } \\
\text { SCHILBEIDAE }\end{array}$ & 1188 & 4.4 & 73.85 & 7.4 \\
\hline 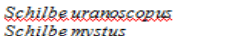 & 2021 & 7.5 & 28.41 & 2.8 \\
\hline $\begin{array}{l}\text { Schilloe vorstus } \\
\text { Rarallia pellucidg }\end{array}$ & $\begin{array}{l}1813 \\
1234\end{array}$ & $\begin{array}{l}6.7 \\
4.6\end{array}$ & $\begin{array}{l}28.09 \\
11.20\end{array}$ & $\begin{array}{l}2.8 \\
1.1\end{array}$ \\
\hline 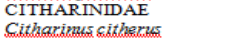 & 1204 & 4.5 & 69.70 & 7.0 \\
\hline $\begin{array}{l}\text { HEPSETIDAE } \\
\text { Hepsetus edoe }\end{array}$ & 1012 & 3.8 & 62.05 & 6.2 \\
\hline ELEOTRIDAE & & & 2130 & \\
\hline Eleotris, senegalensis & $\frac{1052}{26,988}$ & 100 & 1000.57 & $\frac{1.1}{100}$ \\
\hline
\end{tabular}

Table 2: Fish Species caught and their Composition in Agoro-Gbene (Un-dredged Location) Location (June 2009 - May 2011) 


\begin{tabular}{|c|c|c|c|c|}
\hline Family/Species & $\begin{array}{c}\text { No. of } \\
\text { Specimens }\end{array}$ & $\begin{array}{l}\% \text { No. of } \\
\text { Specimen }\end{array}$ & $\begin{array}{l}\text { Weight (Kg) } \\
\text { of Specimens }\end{array}$ & $\begin{array}{r}\% \text { Weight } 0 \\
\text { Specimens }\end{array}$ \\
\hline CHARACIDAE & & & & \\
\hline Bpycinus nurse & 1227 & 3.7 & 88.10 & 4.1 \\
\hline Вирсіичs masreleidotus. & 1133 & 3.4 & 124.88 & 5.7 \\
\hline Hudre surus bxeyis. & 1185 & 3.6 & 112.06 & 5.2 \\
\hline $\begin{array}{l}\text { Alestes baykMgse } \\
\text { MONOCHOKIDAE }\end{array}$ & 1192 & 3.6 & 70.65 & 3.3 \\
\hline Symedantis batenseda & 1271 & 3.8 & 45.61 & 2.1 \\
\hline $\begin{array}{l}\text { Symadontis membxanasceus. } \\
\text { BAGRIDAE }\end{array}$ & 1103 & 3.3 & 63.22 & $\begin{array}{l}2.9 \\
2.8\end{array}$ \\
\hline Clonserichthys furcatus & 1013 & 3.0 & 61.48 & \\
\hline $\begin{array}{l}\text { ClaYRTSE laticeRs, } \\
\text { POL YNEMIIDAE }\end{array}$ & 1032 & 3.1 & 65.69 & 3.0 \\
\hline $\begin{array}{l}\text { Poxdlastulyss quadrifilis, } \\
\text { CIUPEIDAE }\end{array}$ & 1032 & 3.1 & 94.16 & 4.3 \\
\hline $\begin{array}{l}\text { Qdaxathyissa mente } \\
\text { CYNOGLOSSIDAF }\end{array}$ & 1031 & 3.1 & 20.46 & 0.9 \\
\hline $\begin{array}{l}\text { Cynoglassus senegalensis } \\
\text { CYPRINIDAE }\end{array}$ & 828 & 2.5 & 98.53 & 4.5 \\
\hline $\begin{array}{l}\text { Labse squbie, } \\
\text { Labserenesalensis } \\
\text { MORMYRIDAE }\end{array}$ & $\begin{array}{l}1099 \\
1223\end{array}$ & $\begin{array}{l}3.3 \\
3.7\end{array}$ & $\begin{array}{l}115.41 \\
144.01\end{array}$ & $\begin{array}{l}5.3 \\
6.6\end{array}$ \\
\hline 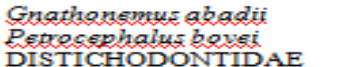 & $\begin{array}{l}1113 \\
1012\end{array}$ & $\begin{array}{l}3.4 \\
3.3\end{array}$ & $\begin{array}{l}87.11 \\
9.35\end{array}$ & $\begin{array}{l}4.0 \\
0.4\end{array}$ \\
\hline 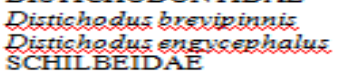 & $\begin{array}{l}1006 \\
1188\end{array}$ & $\begin{array}{l}3.0 \\
3.8\end{array}$ & $\begin{array}{c}206.76 \\
75.92\end{array}$ & $\begin{array}{l}9.5 \\
3.5\end{array}$ \\
\hline Sckills sxanescapus. & 2245 & 6.8 & 23.49 & 1.1 \\
\hline Sckilbs nowrtus & 1927 & 5.8 & 43.99 & 2.0 \\
\hline $\begin{array}{l}\text { Pexalliapellupida } \\
\text { CITHARINIDAE }\end{array}$ & 1273 & 3.8 & 7.66 & 0.4 \\
\hline $\begin{array}{l}\text { Citharinus citherus } \\
\text { HEPSETIDAE }\end{array}$ & 1054 & 3.2 & 145.67 & 6.7 \\
\hline $\begin{array}{l}\text { HeRsetusedoe, } \\
\text { ELEOTRIDAE }\end{array}$ & 1195 & 3.6 & 139.84 & 6.4 \\
\hline $\begin{array}{l}\text { Eleotris, senegalensis } \\
\text { CICHILIDAE }\end{array}$ & 1003 & 3.0 & 27.56 & 1.3 \\
\hline $\begin{array}{l}\text { Hemichromisfaciatus } \\
\text { POL YPTERIDAE }\end{array}$ & 1203 & 3.6 & 39.36 & 1.8 \\
\hline $\begin{array}{l}\text { Pelypterus gnsexpii } \\
\text { NOTOPTERIDAE }\end{array}$ & 1239 & 3.7 & 156.70 & 7.2 \\
\hline Papuresranus afse & 1121 & 3.4 & 51.24 & 2.4 \\
\hline \multirow[t]{2}{*}{ Xenonewstus nieri } & 1109 & 3.3 & 56.60 & 2.6 \\
\hline & 33,275 & 100 & 2175.51 & 100 \\
\hline
\end{tabular}

The LWR was determined following a Log - Log transformation. The Exponential Equation, Degree of Association (r), Coefficient of Determination $\left(\mathrm{r}^{2}\right)$ and significance of Correlation of the Length - Weight relationship for the various fish species in both locations are shown in Tables $3 \& 4$ respectively.. The regression trend indicated that in Ogobiri (Dredged) Location, all fish species exhibited negative allometric weight growth with length exponent (b) ranging from $0.911 \pm 0.01$ (Polydactylus quadrifilis) to $2.709 \pm 0.04$ (Brycinus macrolepidotus) except Schilbe uranoscopus, which exhibited an isometric weight growth with length exponent

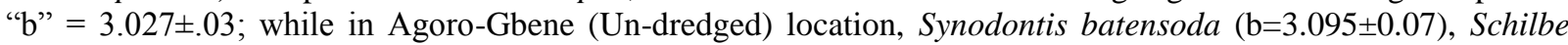
uranoscopus $(\mathrm{b}=3.097 \pm 0.03)$ and Distichodus fasciatus $(\mathrm{b}=3.021 \pm 0.11)$ exhibited isometric growth; others exhibited negative allometric growth with length exponent (b) ranging from $0.777 \pm 0.004$ (Synodoontis membranaceous) to $2.560 \pm 0.04$ (Synoontis nigrita) except Hepsetus odoe which exhibited positive allometric growth $(\mathrm{b}=3.412 \pm \mathrm{a} 0.07)$. The correlation coefficients " $\mathrm{r}$ " ranged from 0.739 (Eleotris senegalensis) to 0.978 (Schilbe mystus) in Ogobiri location and 0.447 (Polypterus ansorgii) to 0.994 (Synodontis membranaceous) in Agoro-Gbene location.

The length exponent (b) ranging from $0.911-3.027$ representing 22 fish species in Ogobiri location and 0.777 - 3.097 representing 28 fish species in Agoro-Gbene location recorded in this study is not in agreement with (b) values ranging from $2.73-3.03$ recorded by Hart and Abowei (2007) for 10 fish species in the Lower Nun River; (b) ranging from $2.790-3.210$ recorded by Fafioye and Oluajo (2005) for 5 fish species in Epe Lagoon, Nigeria; (b) ranging from 2.012 - 2.991recorded by Kumolu-Johnson and Ndimele (2010) for 21 fish species in Ologe Lagoon, Lagos; (b) ranging from 2.719 - 3.580 recorded by Abowei and George (2009) for 5 fish species from Nkoro River, Niger Delta. The correlation coefficient (r) which is the degree of association between length and weight ranging from $0.546-0.956$ in Ogobiri location and $0.336-0.988$ in Agoro-Gbene location is in agreement with $0.951-0.996$ recorded by Hart and Abowei (2007) for 10 fish species in the Lower Nun River. The result from this study shows that in Ogobiri (Dredged) location, the rate of increase in body length is not proportional to the rate of increase in body weight in almost all the fish species except Shilbe uranoscopus; whereas in Agoro-Gbene (Un-dredged) location, the rate of increase in body length is proportional to the rate of increase in body weight in some of the fish species.

Table 3: Length - Weight Regression Equation, Correlation Coefficient (r), Coefficient of Determination $\left(\mathbf{r}^{2}\right)$ and Significance of Correlation for Various Fish Species in Ogobiri Location 


\begin{tabular}{|c|c|c|c|c|}
\hline Fish Species & Regression Equation & $\mathbf{r}$ & $\mathbf{r}^{2}$ & Significance of Correlation \\
\hline Brycinus nurse & $\mathrm{LogW}=0.028+2.648 \mathrm{LogL}$ & 0.940 & 0.884 & $\mathrm{P}<0.05 ; \mathrm{t}=92.41, \mathrm{df}=1119$ \\
\hline Brycinus macrolepidotus & $\mathrm{LOgW}=0.024+2.708 \mathrm{LOgL}$ & 0.894 & 0.799 & $P<0.05 ; t=63.77, d f=1023$ \\
\hline Syndontis balensoda & $\mathrm{LggW}=0.034+2.567 \mathrm{LogL}$ & 0.865 & 0.747 & $\mathrm{P}<0.05 ; \mathrm{t}=58.11, \mathrm{df}=1141$ \\
\hline Syndontis membranaceaus & $\mathrm{LogW}=0.078+2.105 \mathrm{~L} \mathrm{ggL}$ & 0.716 & 0.838 & $P<0.05 ; t=39.23, d f=1209$ \\
\hline Chrysichthys furcatus & $\mathrm{LggW}=0.067+2.183 \mathrm{LQgL}$ & 0.946 & 0.895 & $\mathrm{P}<0.05 ; \mathrm{t}=98.44, \mathrm{df}=1139$ \\
\hline Polydactylus quadrifilis & $\mathrm{LogW}=0.817+0.911 \mathrm{~L} \mathrm{ggL}$ & 0.935 & 0.874 & $\mathrm{P}<0.05 ; \mathrm{t}=90.78, \mathrm{df}=1184$ \\
\hline Odarothrissamento & $\mathrm{LogW}=0.285+1.643 \mathrm{~L} \mathrm{ggL}$ & 0.953 & 0.908 & $\mathrm{P}<0.05 ; \mathrm{t}=106.03, \mathrm{df}=1142$ \\
\hline Paralliapelucida & $\mathrm{LQgW}=0.052+2.245 \mathrm{LQgL}$ & 0.848 & 0.720 & $\mathrm{P}<0.05 ; \mathrm{t}=56.23, \mathrm{df}=1232$ \\
\hline Cynoglossus senegalensis & $\mathrm{LogW}=0.506+0.954 \mathrm{LogL}$ & 0.950 & 0.902 & $\mathrm{P}<0.05 ; \mathrm{t}=107.73, \mathrm{df}=1256$ \\
\hline Hydrocynus brevis & $\mathrm{LogW}=0.018+2.160 \mathrm{LQgL}$ & 0.825 & 0.681 & $\mathrm{P}<0.05 ; \mathrm{t}=51.12, \mathrm{df}=1225$ \\
\hline Labeo coubie & $\mathrm{LOgW}=0.038+2.580 \mathrm{LQgL}$ & 0.967 & 0.935 & $\mathrm{P}<0.05 ; \mathrm{t}=133.01, \mathrm{df}=1230$ \\
\hline Labeo senegalensis & $\mathrm{L} g \mathrm{gW}=0.718+1.802 \mathrm{~L} \mathrm{ggL}$ & 0.952 & 0.907 & $\mathrm{P}<0.05 ; \mathrm{t}=111.89, \mathrm{df}=1284$ \\
\hline Gnathonemus abadii & $\mathrm{LOgW}=0.022+2.616 \mathrm{LQgL}$ & 0.915 & 0.837 & $\mathrm{P}<0.05 ; \mathrm{t}=78.75, \mathrm{df}=1208$ \\
\hline Distichodus brevipinnis & $\mathrm{LOgW}=0.041+2.601 \mathrm{LQgL}$ & 0.972 & 0.945 & $\mathrm{P}<0.05 ; \mathrm{t}=142.69, \mathrm{df}=1186$ \\
\hline Schilbe uranoscopus & $\mathrm{LggW}=0.070+3.027 \mathrm{LggL}$ & 0.918 & 0.842 & $\mathrm{P}<0.05 ; \mathrm{t}=103.78, \mathrm{df}=2019$ \\
\hline Schilbe mystus & $\mathrm{LOgW}=0.032+2.426 \mathrm{LQgL}$ & 0.978 & 0.956 & $\mathrm{P}<0.05 ; \mathrm{t}=198.98, \mathrm{df}=1811$ \\
\hline Citharinus citherus & $\mathrm{LQgW}=0.024 \mathrm{v} 1.983 \mathrm{LggL}$ & 0.780 & 0.609 & $\mathrm{P}<0.05 ; \mathrm{t}=43.25, \mathrm{df}=1202$ \\
\hline Hepsetus odoe & $\mathrm{L} g \mathrm{gW}=0.521+1.632 \mathrm{LggL}$ & 0.935 & 0.874 & $P<0.05 ; t=83.60, d f=1010$ \\
\hline Clarotes laticeps & $\mathrm{LogW}=0.606+2.346 \mathrm{LogL}$ & 0.863 & 0.746 & $P<0.05 ; t=59.16, d f=1194$ \\
\hline Alestes baremose & $\mathrm{LOgW}=0.357+1.621 \mathrm{LQgL}$ & 0.933 & 0.870 & $\mathrm{P}<0.05 ; \mathrm{t}=83.53, \mathrm{df}=1046$ \\
\hline Petrocephalus bovei & $\mathrm{LggW}=0.644+1.165 \mathrm{LggL}$ & 0.933 & 0.870 & $\mathrm{P}<0.05 ; \mathrm{t}=83.08, \mathrm{df}=1034$ \\
\hline Eleotris senegalensis & $\mathrm{LogW}=0.328+1.011 \mathrm{LogL}$ & 0.739 & 0.546 & $\mathrm{P}<0.05 ; \mathrm{t}=35.51, \mathrm{df}=1050$ \\
\hline
\end{tabular}

Table 4: Length - Weight Regression Equation, Correlation Coefficient (r), Coefficient of Determination $\left(\mathbf{r}^{2}\right)$ and Significance of Correlation for Various Fish Species in Agoro - Gbene Location

\begin{tabular}{|c|c|c|c|c|}
\hline Fish Species & Regression Equation & $\mathbf{r}$ & $\mathbf{r}^{2}$ & Significance of Correlation \\
\hline Brycinus nurse & $\mathrm{LggW}=0.285+1.406 \mathrm{LogL}$ & 0.975 & 0.951 & $\mathrm{P}<0.05 ; \mathrm{t}=154.78, \mathrm{df}=1225$ \\
\hline Brycinus macrolepidotus & $\mathrm{LogW}=0.233+1.554 \mathrm{LogL}$ & 0.939 & 0.881 & $\mathrm{P}<0.05 ; \mathrm{t}=91.45, \mathrm{df}=1131$ \\
\hline Syndontis batensoda & $\mathrm{LOgW}=0.020+2.895 \mathrm{LOgL}$ & 0.765 & 0.585 & $\mathrm{P}<0.05 ; \mathrm{t}=86.41, \mathrm{df}=1269$ \\
\hline Syndontis membranaceaus & $\mathrm{LQgW}=0.374+1.156 \mathrm{LogL}$ & 0.994 & 0.988 & $\mathrm{P}<0.05 ; \mathrm{t}=101.82, \mathrm{df}=1101$ \\
\hline Chrysichthys furcatus & $\mathrm{LogW}=0.261+1.997 \mathrm{LogL}$ & 0.986 & 0.972 & $\mathrm{P}<0.05 ; \mathrm{t}=125.31, \mathrm{df}=1011$ \\
\hline Synodontis nigri & $\mathrm{LQgW}=0.046+2.560 \mathrm{LogL}$ & 0.888 & 0.789 & $\mathrm{P}<0.05 ; \mathrm{t}=67.39, \mathrm{df}=1216$ \\
\hline Polydactylus quadrifilis & $\mathrm{LQgW}=0.040+0.770 \mathrm{Log} \mathrm{L}$ & 0.984 & 0.968 & $\mathrm{P}<0.05 ; \mathrm{t}=131.42, \mathrm{df}=1030$ \\
\hline Odacothrissa mento & $\mathrm{L} Q \mathrm{gW}=0.466+1.573 \mathrm{Log} \mathrm{L}$ & 0.991 & 0.981 & $P<0.05 ; t=136.08, d f=1029$ \\
\hline Parallia pelucida & $\mathrm{LogW}=0.582+1.266 \mathrm{LogL}$ & 0.988 & 0.977 & $\mathrm{P}<0.05 ; \mathrm{t}=94.12, \mathrm{df}=1271$ \\
\hline Cynoglossus senegalensis & $\mathrm{L} \circ \mathrm{gW}=0.580+1.097 \mathrm{Log} \mathrm{L}$ & 0.992 & 0.985 & $\mathrm{P}<0.05 ; \mathrm{t}=231.58, \mathrm{df}=826$ \\
\hline Hydrocynus brevis & $\mathrm{LOgW}=0.054+1.943 \mathrm{LogL}$ & 0.965 & 0.931 & $\mathrm{P}<0.05 ; \mathrm{t}=126.92, \mathrm{df}=1183$ \\
\hline Labeo coubie & $\mathrm{L} g \mathrm{gW}=0.033+1.623 \mathrm{LogL}$ & 0.986 & 0.972 & $\mathrm{P}<0.05 ; \mathrm{t}=195.80, \mathrm{df}=1097$ \\
\hline Labeo senegalensis & $\mathrm{LogW}=0.711+1.844 \mathrm{LogL}$ & 0.937 & 0.879 & $P<0.05 ; t=94.08, d f=1221$ \\
\hline Gnathonemus abadii & $\mathrm{LOgW}=0.227+1.392 \operatorname{LogL}$ & 0.976 & 0.952 & $\mathrm{P}<0.05 ; \mathrm{t}=148.49, \mathrm{df}=1111$ \\
\hline Distichodus brevipinnis & $\mathrm{LQgW}=0.103+1.839 \operatorname{LogL}$ & 0.982 & 0.964 & $P<0.05 ; t=163.13, d f=1004$ \\
\hline Schilbe wranoscopus & $\mathrm{LOgW}=0.008+3.077 \mathrm{LogL}$ & 0.915 & 0.833 & $P<0.05 ; t=107.75, d f=2243$ \\
\hline Schilbe mystus & $\mathrm{LogW}=0.131+2.045 \mathrm{LogL}$ & 0.931 & 0.867 & $\mathrm{P}<0.05 ; \mathrm{t}=207.48, \mathrm{df}=1925$ \\
\hline Citharinus citherus & $\mathrm{LogW}=0.741+1.880 \mathrm{LogL}$ & 0.933 & 0.871 & $\mathrm{P}<0.05 ; \mathrm{t}=68.52, \mathrm{df}=1052$ \\
\hline Hepsetus odoe & $\mathrm{LQgW}=0.002+3.412 \mathrm{LogL}$ & 0.820 & 0.673 & $\mathrm{P}<0.05 ; \mathrm{t}=101.72, \mathrm{df}=1193$ \\
\hline Clarotes laticeps & $\mathrm{L} Q \mathrm{gWW}=0.178+2.208 \mathrm{LogL}$ & 0.940 & 0.883 & $\mathrm{P}<0.05 ; \mathrm{t}=84.01, \mathrm{df}=1030$ \\
\hline Alestes baremose & $\mathrm{L} g \mathrm{gW}=0.281+1.965 \mathrm{LogL}$ & 0.961 & 0.923 & $\mathrm{P}<0.05 ; \mathrm{t}=118.42, \mathrm{df}=1190$ \\
\hline Petrocephalus bovei & $\mathrm{LOgW}=0.162+0.884 \operatorname{LogL}$ & 0.981 & 0.963 & $\mathrm{P}<0.05 ; \mathrm{t}=162.23, \mathrm{df}=1010$ \\
\hline Eleotris senegalensis & $\mathrm{LQgW}=0.112+2.355 \operatorname{LogL}$ & 0.962 & 0.926 & $P<0.05 ; t=111.52 ., d f=1001$ \\
\hline Distochodus engycephalus & $\mathrm{LOgW}=0.330+1.830 \mathrm{LogL}$ & 0.702 & 0.492 & $P<0.05 ; t=33.92, d f=1186$ \\
\hline Hemichromus fasciatus & $\mathrm{LogW}=0.027+3.021 \mathrm{LogL}$ & 0.622 & 0.387 & $\mathrm{P}<0.05 ; \mathrm{t}=27.56, \mathrm{df}=1201$ \\
\hline Polypterus ansorgii & $\mathrm{LOgW}=0.262+1.842 \mathrm{LogL}$ & 0.447 & 0.200 & $\mathrm{P}<0.05 ; \mathrm{t}=17.59, \mathrm{df}=1237$ \\
\hline Papyrocranus afer & $\mathrm{LOgW}=0.083+1.308 \mathrm{LogL}$ & 0.984 & 0.968 & $\mathrm{P}<0.05 ; \mathrm{t}=184.32, \mathrm{df}=1119$ \\
\hline Xenomystus nigri & $\mathrm{LogW}=0.466+1.071 \mathrm{LogL}$ & 0.336 & 0.113 & $\mathrm{P}<0.05 ; \mathrm{t}=11.87, \mathrm{df}=1107$ \\
\hline
\end{tabular}

The mean Condition Factor of the various fish species in this study ranged from $0.10-2.24$ in Ogobiri (Dredged) location for 22 fish species and 0.58 - 5.64 in Agoro-Gbene (Undredged) location for 28 fish species. For most species in the present study, condition factor values were higher than the $0.917-0.985$ recorded by Abowei and George (2009) for 5 fish species from Nkoro River, Niger Delta; 0.92 - 0.98 recorded by Hart and Abowei (2007) for 10 fish species in the Lower Nun River; 0.64 - 1.99 recorded by Fafioye and Oluajo (2005) for 5 fish species in Epe Lagoon, Nigeria; 0.12 - 16.29 recorded by Kumolu-Johnson and Ndimele (2010) for 21 fish species in Ologe Lagoon, Lagos. The values obtained for mean Condition Factor and monthly Condition Factor for the various fish species in Agoro-Gbene (Un-dredged) location were significantly higher $(p<0.05)$ than values obtained for Ogobiri (Dredged) location. The statistical analysis of twenty two (22) representative organisms occurring in both locations clearly shows this trend. The mean Condition Factor of the various fish species in Ogobiri (Dredged) location were lesser than those values (2.9 - 4.8) documented by Bagenal and Tesch (1978) for mature fresh water fish. This suggests that the condition of Igbedi creek, upper Nun River around Ogobiri location in comparison to other fresh water bodies is unfavorable to fishes irrespective of season. The results of the Physico-Chemical characteristics of water and sediment samples from this location further confirm this assertion. Water and sediment quality impact can have adverse effect on fisheries resources (Seiyaboh, et. al., (2007). From the results obtained in this study, we can conclude that the fish species in Agoro-Gbene were relatively in a better 
condition than those in Ogobiri location. This might not be unconnected with the dredging operation in Ogobiri location.

\section{Conclusion}

The results indicate that the fish species in the Un-dredged location were generally in a better condition than those in the Dredged location. From the above information, it is concluded that there has been an impact of dredging on the fisheries of Igbedi Creek, Upper Nun River.

Table 5: Condition Factor of Various Fish Species in Ogobiri (June 2009 - May 2011)

\begin{tabular}{|c|c|c|c|c|}
\hline $\begin{array}{l}\text { Fish Species } \\
\end{array}$ & Total No. & Min. & Max. & Mean \pm S.E \\
\hline Brycinus nurse & 1121 & 0.47 & 3.45 & $1.19 \pm 0.01$ \\
\hline Brycinus macrolepidotus & 1025 & 0.12 & 4.10 & $1.13 \pm 0.02$ \\
\hline Syndontis batensoda. & 1143 & 0.28 & 4.44 & $1.16 \pm 0.01$ \\
\hline Syndontis membranaceaus & 1211 & 0.30 & 1.77 & $0.86 \pm 0.01$ \\
\hline Chrysichthus furcatus & 1141 & 0.19 & 3.53 & $0.88 \pm 0.02$ \\
\hline Polvdactylus quadrifilis & 1186 & 0.72 & 5.42 & $2.24 \pm 0.03$ \\
\hline Odaxothrissa mento & 1144 & 0.63 & 1.80 & $1.19 \pm 0.01$ \\
\hline Parallia pelucida & 1234 & 0.36 & 1.78 & $1.21 \pm 0.01$ \\
\hline Cunoglossus senegalensis. & 1258 & 0.23 & 2.11 & $0.81 \pm 0.01$ \\
\hline Hydrocyous brexis. & 1227 & 0.14 & 1.75 & $0.65 \pm 0.01$ \\
\hline Labeo coubie & 1232 & 0.60 & 2.29 & $1.25 \pm 0.01$ \\
\hline Labeo senegalensis & 1286 & 0.55 & 4.09 & $1.47 \pm 0.03$ \\
\hline Gnathonemus abadii & 1210 & 0.11 & 2.05 & $0.84 \pm 0.01$ \\
\hline Distichodus brevipinnis & 1188 & 0.88 & 2.00 & $1.38 \pm 0.01$ \\
\hline Schilbe uranoscopus & 2021 & 0.15 & 1.67 & $0.79 \pm 0.01$ \\
\hline Schilbe mustus. & 1813 & 0.39 & 1.28 & $0.71 \pm 0.004$ \\
\hline Citharinus citherus & 1204 & 0.30 & 4.11 & $1.41 \pm 0.01$ \\
\hline Hepsetus odoe & 1012 & 0.63 & 2.96 & $1.21 \pm 0.02$ \\
\hline Clarotes laticeps. & 1196 & 0.46 & 9.51 & $1.10 \pm 0.02$ \\
\hline Alestes baremose & 1048 & 0.00 & 0.34 & $0.10 \pm 0.003$ \\
\hline Petrocephalus bovei & 1036 & 0.62 & 3.38 & $1.50 \pm 0.02$ \\
\hline Eleotris senegalensis & 1052 & 0.76 & 7.83 & $2.73 \pm 0.05$ \\
\hline
\end{tabular}

Table 6 : Condition Factor of Various Fish Species in Agoro-Gbene (June 2009 - May 2011)

\begin{tabular}{|c|c|c|c|c|}
\hline Fish Species & Total No. & Min. & Max. & Mean \pm S.E \\
\hline Syndontis batensoda & 1271 & 0.77 & 2.62 & $1.57 \pm 0.02$ \\
\hline Chrysichthys furcatus & 1013 & 0.73 & 4.37 & $2.17 \pm 0.93$ \\
\hline Synodontis nigrita & 1218 & 0.23 & 2.30 & $1.53 \pm 0.01$ \\
\hline Polydactylus quadrifilis & 1032 & 0.81 & 6.88 & $3.07 \pm 0.06$ \\
\hline Parallia pelucida & 1273 & 1.00 & 3.90 & $2.01 \pm 0.03$ \\
\hline Cynoglossus senegalensis & 828 & 0.51 & 3.52 & $1.68 \pm 0.03$ \\
\hline Hydrocynus brevis & 1185 & 2.33 & 5.82 & $3.22 \pm 0.03$ \\
\hline Labeo coubie & 1232 & 0.60 & 2.29 & $1.25 \pm 0.01$ \\
\hline Labeo senegalensis & 1286 & 0.55 & 4.09 & $147 \pm 0.03$ \\
\hline Gnathonemus abadii & 1113 & 0.43 & 4.56 & $2.35 \pm 0.04$ \\
\hline Citharinus citherus & 1054 & 1.50 & 5.16 & $2.55 \pm 0.02$ \\
\hline Hepsetus odoe & 1012 & 0.63 & 2.96 & $1.21 \pm 0.02$ \\
\hline Clarotes laticeps & 1032 & 0.61 & 2.68 & $2.18 \pm 0.01$ \\
\hline Alestes baremose & 1192 & 0.39 & 2.53 & $1.70 \pm 0.01$ \\
\hline Petrocephalus bovei & 1012 & 0.90 & 4.98 & $1.95 \pm 0.03$ \\
\hline Eleotris senegalensis & 1003 & 1.52 & 4.59 & $2.59 \pm 0.48$ \\
\hline Distochodus engycephalus & 1188 & 0.19 & 2.87 & $1.26 \pm 0.01$ \\
\hline Hemichromis faciatus & 1203 & 1.08 & 3.98 & $2.34 \pm 0.02$ \\
\hline Polypterus ansorgii & 1239 & 0.29 & 0.91 & $0.58 \pm 0.003$ \\
\hline Papyrocraus afer & 1121 & 0.52 & 2.45 & $1.11 \pm 0.02$ \\
\hline Xenomystus nigri & 1109 & 1.00 & 3.47 & $1.24 \pm 0.45$ \\
\hline
\end{tabular}

KEY: Min. = Minimum, Max. = Maximum, S. E. = Standard Error 


\section{References}

[1] Abowei, J. F. N. (2000): Aspects of the fisheries of the lower Nun River. Ph.D. Dissertation, University of Port Harcourt. 180pp.

[2] Abowei, J. F. N. and George, A. D. I. (2009). Some physical and chemical characteristics in Okpoka Creek, Niger Delta, Nigeria. Research Journal of Environmental and Earth Sciences 1(2):45-53.

[3] Alfred-Ockiya, J. F. (1983) Field characteristics of some common fresh water fishes of the Niger Delta, Nigeria. Unpublished Department paper. No. 3. 36pp.

[4] Ault, J. S., Bohnsack, B.A., and Meester, G.A. (1998). A retrospective (1979-1996) multispecies assessment of coral reef fish stocks in Florida Keys. Fishery $\backslash$ Bulletin 96(3): $\quad 395-414$.

[5] Bagenal, T. B. and Tesch, A. T. (1978). Conditions and Growth Patterns in Fresh Water Habitats. Blackwell Scientific Publications, Oxford, pp: 75-89.

[6] Boseman, M. (1963) Annotated list of fishes from the Niger Delta. Zool. Verh. Lediden 61: 1- 48

[7] Clark, S. H. (1979): Application of Bottom Trawl Surve. Fisheries 4 (3): 9 - 15.

[8] Daget, J. (1954): Less poisons du Niger Superieur - Bul. L. I. F. A. N. 36:1-391.

[9] Fafioye, O.O. and Oluajo, O.A. (2005) Length-weight relationships of five fish species in Epe Lagoon, Nigeria. African journal of Biotechnology, 4 (7). --. 749-751 ISSN 1684-5315.

[10] Gayando, F. C. Jr. and Pauly, D. (1997) eds. FAO-ICLARM Stock Assessment Tools (FiSAT) Reference manual FAO Computerised Information series (Fisheries) No. 8 Rome, FAO, 262p.

[11] Gebhards, S. (1973). Effects of channelization on fish. In Panel of Stream Channelization and Trout Fisheries (White, R.J. ed.). Trout. 14.

[12] Hart, A. I. and Zabbey, N. (2005). Physico-chemical and benthic fauna of Woji Creek in the Lower Niger Delta, Nigeria. Environ. Ecol., 23(2): 361-368.

[13] Hart, A. I. and Abowei, J. F. N. (2007). A study of the length-weight relationship, condition factor and age of ten fish species from the lower Nun River. Niger Delta. Afr. J. Appl. Z Zool. Environ. Biol., 9:13-19.

[14] Holden, M. and Reed, W. (1972). West African Fresh water fishes. Longmans Limited London 33p.

[15] Jiri, C. (1976). A colour guide of familiar fresh water fishes. Octopus Books Ltd. London 25p.

[16] Karr, J.R. (1981). Assessment of biotic integrity using fish communities. Fisheries 6:21-27.

[17] Loveque, C., Pyugy, O. and Teugels, G. G ed (1991). The fresh and brackish water fishes of West Africa. Vol. 1 Musee Royale de. 1 Afrique Centrale. Tervurem, Belgique, Editions de IORSTOM: 384 p.

[18] Otobo, A. J. T. (1981) Identification of fish species in a stretch of River Nun. HND Project Rivers State University of Science \& Technology, Port Harcourt.

[19] Pauly, D. (1983). Some simple methods for the assessment of tropical fish stock FAO Fish Tech. paper No. 234. 52p.

[20] Poll, M. (1974): Synopsis and Geographical Distribution of the clupeidea in Africa Firewater. Distribution of three New species. Bull. De Ca Class Des Sci: 5 Sere Tom LX(2)141-161

[21] Reine, K., and Clarke, D.(1998). Entrainment by hydraulic dredges - A review of potential impacts. Technical Note Dredging Operations and Environmental Research (DOER)-E1. U.S. Army Engineer Research and Development Center, Vicksburg, MS

[22] Reed, W., Burchard, J., Jennes, J. and Yaro I., (1967). Fish and fisheries of Northern Nigeria MOA 226pp. [AQF] [FISR]

[23] Reid, G.M. and Sydenham, H. (1978). A check list of lower Benue River Fishes and on ichtho-geographical review of the Benue River. J. Nat Hist 13(1) 41-67.

[24] Seyaboh, E.I., Ekweozor, I.K.E., Otobo, A.J.T. and Ogamba, E.N. (2007) Effect of Tombia Bridge Construction on the Fisheries of Nun River iin Central Niger Delta. World Journal of Biotechnology. 8 (1) : 1272-1279.

[25] Sikoki, F.D., Hart, A.I. \& Abowei, F. (1998) Gill-net Selectivity and Fish Abundance in the lower Nun River, Nigeria. Journal of Applied Science and Environmental Management. Vol. 1, No. 1 pp 13-19.

[26] Whitehead, P. J. P. (1984). Family clupeidae Daget, J. Gosse, J. P. Thys. Van den. Audennerede, D. F. S (eds). Checklist of the freshwater fishes of Africa vol. 1 ORSTOM: 11-20.

[27] Whyte, S,A, (1975) Distribution, trophic relationship and breeding habits of the fish Busumtwi, Ghana. J. Zool. Lond. 177:25 